\title{
Scalar sextet in the 331 model with right-handed neutrinos
}

\author{
Nguyen Anh Ky and Nguyen Thi Hong Van \\ Institute of Physics and Electronics, 10 Dao Tan, Hanoi, Vietnam
}

\begin{abstract}
A Higgs sextet is introduced in order to generate Dirac and Majorana neutrino masses in the 331 model with right-handed neutrinos. As will be seen, the present sextet introduction leads to a rich neutrino mass structure. The smallness of neutrino masses can be achieved via, for example, a seesaw limit. The fact that the masses of the charged leptons are not effected by their new Yukawa couplings to the sextet is convenient for generating small neutrino masses.
\end{abstract}

PACS numbers: 12.10.Dm, 12.60.Fr, 14.60.Pq

\section{INTRODUCTION}

The 331 model 1, 2, 3, 4] based on the gauge group $S U(3)_{C} \otimes S U(3)_{L} \otimes U(1)$, is one of the interesting extensions of the standard model (SM). Firstly, it is stated that the model guarantees the number of the families to be three. Secondly, the Peccei-Quinn symmetry [5]-a solution of the strong CP problem, naturally occurs in the 331 model 6 . The third reason making this model interesting is that one of the quark families transforms under the gauge group differently from the other two. This could naturally explain unbalancing heavy top quarks in the fermion mass hierarchy, deviations of $A_{b}$ from the SM prediction, etc. Recent analyzes indicated that signals of new particles in this model, i.e., bileptons [7, 8] and exotic quarks [9], may be observed at the Tevatron and the LHC. The fact that the 331 model predicts not very high new mass scales, at the order of a few TeV's only [10], may help us to solve in a near future some hard problems like the $g-2$ problem 8], neutrino oscillations and masses (see, for example, [1] -16$]$ ), etc..

The 331 model has several versions. Two of them are the minimal version [1, 2, 3] (in which no new lepton is introduced and all leptons of each generation are grouped in one triplet), and the version with righthanded neutrinos (RHN's) [4]. Each of the two versions has specific feactures but at the beginning they both were introduced with a Higgs sector consisting of three scalar $S U(3)_{L}$-triplets (or shortly, triplets) only. Later, a Higgs $S U(3)_{L}$-sextet (or shortly, a sextet) described by a symmetric tensor $S_{i j}=S_{j i}, i, j=1,2,3$, was added to the minimal version in order to properly generate all charged lepton masses [3]. In the version with RHN's, so far, however, a Higg sextet has not been needed as the three Higgs triplets have been enough for generating masses for all charged leptons and quarks. As far as neutrinos are concerned, we can make them remaining massless by choosing an appropriate configuration of vacuum expectation values (VEV's) of the Higgs sectors. The problem related to fermion masses seems quite simple here. Both the theoretical motivations and the experimental results in the last time 17] - 34], however, give more and more evidences supporting the idea of massive neutrinos which may be of a Dirac or a Majorana type. So far, many theoretical mechanisms for generating neutrino masses have been suggested (see, for example, 33] - 40] and references therein). In the models with massive Dirac, not Majorana, neutrinos, a certain combination of the lepton numbers can be a conserved quantity, while an existence of massive Majorana neutrinos violates it. So, the question of massive neutrinos of which type is directly related to a fundamental question of symmetries in particle physics. In the 331 models there have been several ways neutrinos to get masses.

The minimal 331 model can generate Majorana masses which could be tree-level (if a non-zero VEV of the sextet component $S_{00} \equiv \sigma_{1}^{0}$ is allowed) [11] or radiatively induced 12, 13]. There are also other mechanisms for generating neutrino masses for this version of the 331 model, for example, in 15. the sextet is replaced by a neutral scalar singlet and a dimension-seven effective operator is introduced. Experimental and practical data show that the neutrinos if massive have very tine masses (only a few eV's or less). In the frameworks of the minimal 331 model some attempts for explaining the smallness of neutrinos masses have been made (see, for eaxmple, 11 - 15]). As far as the 331 model with RHN's is concerned, investigations on neutrinos masses are much poorer here. In this model, the neutrinos (more precisely, two of them) can gain Dirac masses if a definite one of the three Higgs triplets develops a non-zero VEV but at the present, as stated in [4], it is not known how to get small neutrino masses. Additionally, there is no explicit evidence for the Majorana neutrino masses to be excluded in advance from consideration when the lepton number has no real meaning in both versions of the 331 model (as a lepton and its antiparticle are simultaneosly components of one and the same multiplet) and when neutrinoless double beta $\left((\beta \beta)_{0 \nu}\right)$ decays (which are sensitive to the existence of Majorana neutrinos and violate the total lepton number by two uinits) are still considered as possible processes 21] - 26]. Moreover, the presence of the right-handed neutrinos in the theory is a good reason for considering neutrino masses of Majorana type. In this paper we show that Majorana neutrino masses can be introduced in the 331 model with RHN's by adding a scalar sextet to the Higgs sector. 
Beside Majorana neutrino masses, this modified model allows Dirac neutrino masses too. The smallness of the neutrino masses can be achieved by taking, for example, a seesaw limit. In general, as will be seen, the sextet introduction suggests a rich structure of the neutrino masses. The present paper is organized as follows.

In the next section we briefly recall some necessary elements of two versions of the 331 model, namely the minimal version and the original version with RHN's in which the Higgs sector contains only three scalar triplets. A scalar sextet is introduced to this sector in the Section 3 where we show how neutrino masses can be generated after the sextet introduction. The conclusion and some comments are given in the last section, Section 4.

\section{SOME ELEMENTS OF THE 331 MODELS}

\section{A. The minimal version}

Let us start with the minimal version of the 331 model [1, 2, 3]. The leptons of all generations transform under the gauge group $S U(3)_{C} \otimes S U(3)_{L} \otimes U(1)$ (or the 331-gauge group, for short) in one and the same way as follows

$$
f_{L}^{a}=\left(\begin{array}{c}
\nu_{L}^{a} \\
l_{L}^{a} \\
\left(l_{R}^{a}\right)^{c}
\end{array}\right) \sim(1,3,0), \quad l_{R}^{c} \equiv\left(l_{R}\right)^{c},
$$

where $a=1,2,3$, is a generation index. The Higgs sector of the minimal 331 model consists of three triplets and one sextet which ensure masses for all fermions in the model via symmetry breaking which could follow the order

$$
\begin{gathered}
S U(3)_{C} \otimes S U(3)_{L} \otimes U(1)_{N} \\
\langle\chi\rangle \downarrow \\
S U(3)_{C} \otimes S U(2)_{L} \otimes U(1)_{Y} \\
\langle\rho\rangle,\langle\eta\rangle \downarrow\langle S\rangle \\
S U(3)_{C} \otimes U(1)_{Q},
\end{gathered}
$$

where $\langle\chi\rangle,\langle\rho\rangle,\langle\eta\rangle$ and $\langle S\rangle$ are VEV's of the Higgs fields $\chi, \rho, \eta$ and $S$, rescpectively (see [3, 41] for more details). As far as the quark sector is concerned, one of the quark generations transforms differently from the other two which in turn transform in one and the same way under the 331-gauge group.

\section{B. The version with right-handed neutrinos}

In the original version of the 331 model with RHN's proposed in [4] a lepton multiplet of each generation, obtained from the corresponding one in (II) by replacing the third component $l_{R}$ with a right-handed neutrino $\nu_{R}$, tranforms under the 331-gauge group as

$$
f_{L}^{a}=\left(\begin{array}{c}
\nu_{L}^{a} \\
l_{L}^{a} \\
\left(\nu_{R}^{c}\right)^{a}
\end{array}\right) \sim(1,3,-1 / 3), l_{R}^{a} \sim(1,1,-1),
$$

where $a$ is a generation index. In our opinion, the righthanded neurtino on the third component of $f_{L}$ could be in general different from the anti-neutrino $\nu_{R}$ - the antiparticle of the neutrino $\nu$, staying on the first component of $f_{L}$. To avoid any confusion, instead of $\nu_{R}^{c}$ (used in [4]) we suggest [42] another notation, say $N_{R}^{c}$, for the third component of $f_{L}$ :

$$
f_{L}^{a}=\left(\begin{array}{c}
\nu_{L}^{a} \\
l_{L}^{a} \\
\left(N_{R}^{c}\right)^{a}
\end{array}\right) \sim(1,3,-1 / 3), l_{R}^{a} \sim(1,1,-1) .
$$

Here, as in the minimal version, two of the quark generations transfrom in one and the same way, while the remaining one transforms differently (as the quark sector is not considered in this paper, its explicit stucture and transformation are not given here).

The Higgs sector of the original version with RHN's [4] consists of three scalar triplets

$\eta=\left(\begin{array}{c}\eta_{1}^{0} \\ \eta_{2}^{-} \\ \eta_{3}^{0}\end{array}\right) \sim(1,3,-1 / 3), \quad \rho=\left(\begin{array}{c}\rho_{1}^{+} \\ \rho_{2}^{0} \\ \rho_{3}^{+}\end{array}\right) \sim(1,3,2 / 3)$
$\chi=\left(\begin{array}{c}\chi_{1}^{0} \\ \chi_{2}^{-} \\ \chi_{3}^{0}\end{array}\right) \sim(1,3,-1 / 3)$

The Yukawa couplings in this case

$$
\begin{aligned}
\mathcal{L}_{y u k}^{\chi}= & \lambda_{1} \bar{Q}_{1 L} u_{1 R}^{\prime} \chi+\lambda_{2 i j} \bar{Q}_{i L} d_{j R}^{\prime} \chi^{\dagger}+H . c ., \\
\mathcal{L}_{Y u k}^{\rho}= & \lambda_{1 a} \bar{Q}_{1 L} d_{a R} \rho+\lambda_{2 i a} \bar{Q}_{i L} u_{a R} \rho^{\dagger} \\
& +G_{a b} \bar{f}_{L}^{a}\left(f_{L}^{b}\right)^{c} \rho^{\dagger}+G_{a b}^{\prime} \bar{f}_{L}^{a} e_{R}^{b} \rho+H . c ., \\
\mathcal{L}_{y u k}^{\eta}= & \lambda_{3 a} \bar{Q}_{1 L} u_{a R} \eta+\lambda_{4 i a} \bar{Q}_{i L} d_{a R} \eta^{\dagger}+H . c .,
\end{aligned}
$$

where $a, b=1,2,3, i=2,3$, can ensure (via the LHS symmetry breaking scheme in (2)) masses for all quarks and charged leptons as well as Dirac masses for two of the neutrinos [4]. This model, however, cannot explain the smallness of neutrino masses and does not generate (at least, at the tree-level) a Majorana neutrino mass which, as discussed above, is by no reason to be exluded in advance from consideration. This problem may be solved by introducing a scalar sextet to the model. 


\section{SEXTET AND NEUTRINO MASSES IN THE 331 MODEL WITH RHN'S}

A neutrino mass (at the tree level) can be generated by coupling an appropariate Higgs boson to $\bar{f}_{L}\left(f_{L}\right)^{c}$. As an $S U(3)_{L}$-tensor the latter is a (tensor) product $\mathbf{3}^{*} \otimes \mathbf{3}^{*}$ of two $S U(3)_{L}$-anti-triplet $\bar{f}_{L}$ and $\left(f_{L}\right)^{c}$, consequently, it can be decomposed into a direct sum of a triplet 3 (the anti-symmetric part of the tensor) and an anti-sextet $\mathbf{6}^{*}$ (the symmetric part of the tensor):

$$
\mathbf{3}^{*} \otimes \mathbf{3}^{*}=\mathbf{3} \oplus \mathbf{6}^{*} \text {. }
$$

To constitute an $S U(3)_{L}$-invariant quantity we can contract $\bar{f}_{L}\left(f_{L}\right)^{c}$ with an anti-triplet $\mathbf{3}^{*}$ and/or a sextet 6. The term $G_{a b} \bar{f}_{L}^{a}\left(f_{L}^{b}\right)^{c} \rho^{\dagger}$ in the Yukawa Lagrangians (5) is a contraction of the first kind. Here, three $S U(3)_{L^{-}}$ indeces are antisymmetrized by contracting with the anti-symmetric $S U(3)_{L}$-tensor $\epsilon_{i j k}$, i.e., the rank-two tensor $\bar{f}_{L}\left(f_{L}\right)^{c}$ is anti-symmetrized (therefore, it transforms under $S U(3)_{L}$ as a triplet 3 ) and contracted with $\rho^{\dagger}$, an anti-triplet $\left(\mathbf{3}^{*}\right)$. At $\langle\rho\rangle \neq 0$, the above Yukawa coupling term can generate Dirac masses for two of the three neutrinos (while the third one remains massless) 4]. This way for generating neutrino masses, however, gives no indication for that the neutrino masses obtained are small, and it excludes the Majorana neutrino masses which (as explained above) might be important. A scalar sextet added to the Higgs sector may solve this questions.

A scalar field tranforming under $S U(3)_{L}$ as a sextet 6 can be described by a symmetric tensor which in the present case has the following explicit form and 331gauge transformation law

$$
\mathcal{S}=\left(\begin{array}{ccc}
\tau_{1}^{0} & T_{1}^{-} / \sqrt{2} & \tau_{2}^{0} / \sqrt{2} \\
T_{1}^{-} / \sqrt{2} & T_{2}^{--} & T_{3}^{-} \\
\tau_{2}^{0} / \sqrt{2} & T_{3}^{-} & \tau_{3}^{0}
\end{array}\right) \sim(1,6,-2 / 3)
$$

A non-zero VEV of this sextet $\mathcal{S}$ coupled to the symmetric part of $\bar{f}_{L}\left(f_{L}\right)^{c}$ could give rice to Dirac and/or Majorana neutrino masses without effecting the masses of the charged leptons. The Lagrangian term corresponding to this Yukawa coupling is

$$
G_{a b}^{s} \bar{f}_{L}^{a}\left(f_{L}^{b}\right)^{c} \mathcal{S}+\text { H. c., }
$$

where $G_{a b}^{s}$ are new coupling constants; $a, b=1,2,3$, are generation indeces, while the $S U(3)_{L}$-indeces are omitted. A general structure of a $\mathrm{VEV}$ of $\mathcal{S}$ could be

$$
\langle\mathcal{S}\rangle=\left(\begin{array}{ccc}
\omega_{1} & 0 & \omega_{2} / \sqrt{2} \\
0 & 0 & 0 \\
\omega_{2} / \sqrt{2} & 0 & \omega_{3}
\end{array}\right)
$$

where $\omega_{n}$ are VEV's of the neutral sextet components $\tau_{n}^{0}$, $n=1,2,3$. This $\mathrm{VEV}$ when inserted in (7) leads to the mass term

$$
G_{a b}^{s}\left(\bar{\nu}_{L}^{a}, \bar{l}_{L}^{a},\left(\bar{N}_{R}^{c}\right)^{a}\right)\left(\begin{array}{ccc}
\omega_{1} & 0 & \omega_{2} / \sqrt{2} \\
0 & 0 & 0 \\
\omega_{2} / \sqrt{2} & 0 & \omega_{3}
\end{array}\right)\left(\begin{array}{c}
\left(\nu_{L}^{c}\right)^{b} \\
\left(l_{L}^{c}\right)^{b} \\
N_{R}^{b}
\end{array}\right)
$$

which in the neutrino subspace has the form

$$
G_{a b}^{s}\left(\bar{\nu}_{L}^{a},\left(\bar{N}_{R}^{c}\right)^{a}\right)\left(\begin{array}{cc}
\omega_{1} & \omega_{2} / \sqrt{2} \\
\omega_{2} / \sqrt{2} & \omega_{3}
\end{array}\right)\left(\begin{array}{c}
\left(\nu_{L}^{c}\right)^{b} \\
N_{R}^{b}
\end{array}\right)
$$

The latter is nothing but the familiar Dirac-Majorana mass term

$$
\frac{1}{2}\left(\bar{\nu}_{L}, \bar{N}_{R}^{c}\right)\left(\begin{array}{cc}
\mathbf{m}_{T} & \mathbf{m}_{D} \\
\mathbf{m}_{D} & \mathbf{m}_{S}
\end{array}\right)\left(\begin{array}{c}
\nu_{L}^{c} \\
N_{R}
\end{array}\right)
$$

where the generation indeces are omitted and $\mathbf{m}_{T, D, S}$ are $3 \times 3$ matrices with the following elements

$$
\begin{aligned}
& \left(\mathbf{m}_{T}\right)_{a b}=2 G_{a b}^{s} \omega_{1}, \quad\left(\mathbf{m}_{D}\right)_{a b}=\sqrt{2} G_{a b}^{s} \omega_{2}, \\
& \left(\mathbf{m}_{S}\right)_{a b}=2 G_{a b}^{s} \omega_{3} .
\end{aligned}
$$

An analysis of a mass term of this kind is well known. For example, at the seesaw limit

$$
\mathbf{m}_{T} \approx 0, \quad \mathbf{m}_{S} \gg \mathbf{m}_{D}
$$

or equivalently,

$$
\omega_{1} \approx 0, \quad \omega_{3} \gg \omega_{2}
$$

we get two eigen mass matrices (generation-mixing, in general)

$$
\mathbf{m}_{1}=\left(\mathbf{m}_{D}\right)^{T}\left(\mathbf{m}_{S}\right)^{-1} \mathbf{m}_{D}, \quad \mathbf{m}_{2}=\mathbf{m}_{S} .
$$

The condition (14) could be accepted in some circumstance as $\omega_{3}$ characterizes the energy scale of bearking $S U(3)$ down to $S U(2)$ and therefore it must be much bigger than $\omega_{2}$ and $\omega_{1}$ characterizing the scales of breaking $S U(2)$ and $U(1)$ :

$$
\omega_{3} \gg \omega_{2} \gg \omega_{1} \text {. }
$$

Diagonalizing the matrix $G_{a b}^{s}$ and keepping (14) we get for each eigenstate of $G_{a b}^{s}$, a diagonalized mass matrix,

$$
\begin{aligned}
& \left(m_{T}\right)_{k}=2 G_{k}^{s} \omega_{1}, \quad\left(m_{D}\right)_{k}=2 G_{k}^{s} \omega_{2} / \sqrt{2}, \\
& \left(m_{S}\right)_{k}=2 G_{k}^{s} \omega_{3}, \quad k=1,2,3,
\end{aligned}
$$

leading to the Majorana neutrinos

$$
\begin{aligned}
& \left(n_{1 L}\right)_{k}=\left(\nu_{L}\right)_{k}-\frac{\left(m_{D}\right)_{k}}{\left(m_{S}\right)_{k}}\left(N_{R}^{c}\right)_{k}, \\
& \left(n_{2 L}\right)_{k}=\frac{\left(m_{D}\right)_{k}}{\left(m_{S}\right)_{k}}\left(\nu_{L}\right)_{k}+\left(N_{R}^{c}\right)_{k},
\end{aligned}
$$


with masses

$$
\begin{aligned}
& \left(m_{1}\right)_{k}=\left(m_{D}^{2}\right)_{k} /\left(m_{S}\right)_{k} \equiv G_{k}^{s} \frac{\left(\omega_{2}\right)^{2}}{\omega_{3}} \\
& \left(m_{2}\right)_{k}=\left(m_{S}\right)_{k} \equiv 2 G_{k}^{s} \omega_{3}
\end{aligned}
$$

where $(M)_{k}$ is a diagonal element of a diagonalized matrix M. An estimation of these masses can be made by taking bounds of the VEV's $\omega_{i}$ and the coupling constant $G^{s}$ via analyzing different processes (decay modes and branching ratios) and an appropriate scalar potential in the Higgs sector with participation of the sextet. However, basing on the experimental data, theoretical imagination or assumption and some trick (the ratio $m_{1} / m_{2}=\left(\omega_{2} / \omega_{3}\right)^{2}$ does not depend on the coupling constants $G$ ) we can avoid this, a bit long, procedure.

If we can neglect the generation mixing we should have, for each generation, Majorana neutrinos

$$
\begin{aligned}
\left(n_{1 L}\right)_{a} & =\left(\nu_{L}\right)_{a}-\frac{\left(m_{D}\right)_{a}}{\left(m_{S}\right)_{a}}\left(N_{R}^{c}\right)_{a} \\
\left(n_{2 L}\right)_{a} & =\frac{\left(m_{D}\right)_{a}}{\left(m_{S}\right)_{a}}\left(\nu_{L}\right)_{a}+\left(N_{R}^{c}\right)_{a}
\end{aligned}
$$

with masses

$$
\begin{aligned}
& \left(m_{1}\right)_{a}=\left(m_{D}^{2}\right)_{a} /\left(m_{S}\right)_{a} \equiv G_{a}^{s} \frac{\left(\omega_{2}\right)^{2}}{\omega_{3}} \\
& \left(m_{2}\right)_{a}=\left(m_{S}\right)_{a} \equiv 2 G_{a}^{s} \omega_{3}
\end{aligned}
$$

respectively. If the magnitude of $m_{D}$ is in the order of those of the charged lepton masses, i.e., $\left(m_{D}\right)_{1} \sim m_{e}$, $\left(m_{D}\right)_{2} \sim m_{\mu},\left(m_{D}\right)_{3} \sim m_{\tau}$, while $m_{S}$ is around $1 \mathrm{TeV}$ or bigger [8, 10], we get the following estimation on the masses of neutrinos $\nu_{e}, \nu_{\mu}$ and $\nu_{\tau}$ :

$$
m_{\nu_{e}}<0.25 \mathrm{eV}, m_{\nu_{\mu}}<11.2 \mathrm{KeV}, m_{\nu_{\tau}}<3.16 \mathrm{MeV}
$$

It is some thing like the normal hierarchy of neutrino masses. A popular theoretical assumption (for a seesaw model), however, says that $m_{S}$ related to the mass of a right-handed neutrino could be very large, with a magnitude near to that of $M_{G U T}$. A value of $m_{S}$ between the range $10^{10} \mathrm{GeV}<m_{S}<10^{15} \mathrm{GeV}$ can ensure the neutrino masses very small even for relatively large $m_{D}$ as accepted in some models (e.g., $m_{D} \sim 200 \mathrm{GeV}$ in [31]).

Let us return to Eqs. (17) - (21) since the generation mixing is already a real fact [17]-[20]. According to the recent data 27] - 34] giving an upper bound of neutrino masses in the order of $1 \mathrm{eV}$ (or less) we get a relation between the magnitudes of $m_{D}$ and those of $m_{S}$ :

$$
\frac{m_{D}^{2}}{m_{S}}=10^{-9} \mathrm{GeV}
$$

from which if we take the bound $300 \mathrm{GeV}<m_{S}<1 \mathrm{TeV}$ [8, 10] we should have

$$
0.548 \mathrm{MeV}<m_{D}<1 \mathrm{MeV}
$$

The latter bound is around the electron mass. As mentioned above the ratio $m_{1} / m_{2}$,

$$
\frac{m_{1}}{m_{2}}=\left(\frac{\omega_{2}}{\omega_{3}}\right)^{2}
$$

is somewhat "universal", that is, it is independent from the coupling constants but the ratio $\omega_{2} / \omega_{3}$. Thus, a ratio can be predicted if the other one is known.

In a standard way 35] - 40] we can consider other limits of the mass term (11): the pure Majorana limit $\left(m_{D}=0\right)$, the Dirac limit $\left(m_{T}=m_{S}=0\right)$ or the pseudoDirac limit (when $m_{T} \ll m_{D}$ and $m_{S} \ll m_{D}$ ), etc. In the latter case, there are two almost-degenerate Majorana neutrinos with a very small mass spliting. For $m_{S}=0$, $m_{T} \ll m_{D}$ the masses are $m_{ \pm}=m_{D} \pm m_{T} / 2$. Another case is when both $m_{D}$ and $m_{S}$ (and/or $m_{T}$ ) are very small and comparable. This case leads to non-degenerate Majorana neutrinos and, as in the pseudo-Dirac case, to signicant ordinary-sterile $\left(\nu_{L}^{a}-\left(N^{c}\right)_{L}^{a}\right)$ mixings (to be or not to be confirmed by LSND). However, the latest counted cases may not be compatible with the symmetry breaking hierarchy of the model.

\section{CONCLUSION}

We have introduced in the 331 model with RHN's a new Higgs field, a sextet under $S U(3)_{L}$, with the hope to generate proper neutrino masses. In the original version of the model [4], the neutrinos, more precisely, two of them, can have Dirac masses only but the presence of the right-handed neutrinos in the model indicates that it is possible and reasonable to include Majorana neutrino masses, especially, when the processes like $(\beta \beta)_{0 \nu}$ decays are still considered to be possible. The sextet introduction, suggesting a rich neutrino mass structure, may solve this problem and generate neutrino masses in a right order. Next, following [41] we can investigate the mass spectra of this extended scalar sector of the 331 model with RHN's (thus we need a notion of the lepton number for the case and follows [1] to construct a lepton-number-conserved potential).

Note added: Some earlier versions with two neutrinos in a lepton triplet of the 331 model were considered in [43, 44, 45]. A Higgs sextet was introduced and discussed briefly in 44, 45]. However, the corresponding neutrino mass term was analyzed in other aspects, its seesaw limit, therefore, the seesaw mechanism, was not considered and Majorana neutrinos and masses were almost neglected in [44, 45] (due to the circumstance in their epoch), unlike in the present paper. 


\section{Acknowledgments}

We would like to thank the referee for drawing our attention to the works [43, 44, 45] giving us interesting information. One of us (N.A.K.) would like to thank S. Petcov and A. Smirnov for discussions and H.N. Long for the cooperation in doing earlier works on the 331 model which are useful for the present work. We also would like to thank S. Randjbar-Daemi for kind hospitality at the Abdus Salam International Centre for Theoretical Physics, Trieste, Italy.

This work was supported in part by the National Research Program for Natural Sciences of Vietnam under Grant No 410804.
[1] F. Pisano and V. Pleitez, Phys. Rev. D 46, 410 (1992).

[2] P. H. Frampton, Phys. Rev. Lett. 69, 2889 (1992).

[3] R. Foot, O. F. Hernandez, F. Pisano and V. Pleitez, Phys. Rev. D 47, 4158 (1993).

[4] R. Foot, H. N. Long and Tuan A. Tran, Phys. Rev. D 50, R43 (1994).

[5] R. D. Peccei and H. R. Quinn, Phys. Rev. Lett. 38, 1440 (1977); Phys. Rev. D 16, 1791 (1977).

[6] P. B. Pal, Phys. Rev. D 52, 1659 (1995).

[7] B. Dion, T. Gregoire, D. London, L. Marleau and H. Nadeau, Phys. Rev. D 59, 075006 (1999).

[8] Nguyen Anh Ky, Hoang Ngoc Long and Dang Van Soa, Phys. Lett. B486, 140 (2000); Nguyen Anh Ky and Hoang Ngoc Long, "The anomalous magnetic moment of muon: from the E821 experiment to bilepton masses", hep-ph/0103247

[9] P. Das, P. Jain, and D. W. McKay, Phys. Rev. D 59, 055011 (1999); Y. A. Coutinho, P. P. QueirozFilho and M. D. Tonasse, Phys. Rev. D 60, 115001 (1999).

[10] D. Ng, Phys. Rev. D 49, 4805 (1994).

[11] , M. B. Tully and G. C. Joshi, Phys. Rev. D 64, 011301(R) (2001).

[12] Y. Okamoto and M. Yasue, Phys. Lett. B466, 267 (1999).

[13] T. Kitabayashi and M. Yasue, Phys. Lett. B508, 85 (2001); Phys. Rev. D 63, 095002 (2001).

[14] J. C. Montero, C. A. deS. Pires and V. Pleitez, Phys. Lett. B502, 167 (2001).

[15] J. C. Montero, C. A. deS. Pires and V. Pleitez, Phys. Rev. D 65, 093017 (2002).

[16] A. Gusso, C. A. de S. Pires and P. S. Rodrigues da Silva, Mod. Phys. Lett. A18, 1849 (2003).

[17] Super-Kamiokande collaboration, Y. Fukuda et al., Phys. Rev. Lett. 81, 1562 (1998); Phys. Lett. B436, 33 (1998); Phys. Lett. B433, 9 (1998).

[18] Soudan 2 collaboration, W. W. Allison et al., Phys. Lett. B449, 137 (1999).

[19] SNO collaboration, Q. R. Ahmad et al., Phys. Rev. Lett. 89, 011301 (2002); ibid., 011302 (2002); V. Barger et al., Phys. Lett. B537, 179 (2002); A. Baudyopadhyay et al., Phys. Lett. B540, 14 (2002); J. Barcall et al., JHEP 0207, 054 (2002); G. L. Fogli, E. Lisi, A. Marrone, D. Montanino and A. Palazzo, Phys. Rev. D 66, 053010 (2002); B. Aharmim et al, nucl-ex/0502021

[20] LSND collaboration, C. Athanassopoulos et al, Phys. Rev. Lett. 81, 1774 (1998); Phys. Rev. C 58, 2489 (1998).

[21] Yu. Zdesenko, Rev. Mod. Phys. 74, 663 (2002).

[22] C. Aalseth et al, Phys. Atom. Nucl. 67, 2002 (2004).

[23] S. Petcov, "Theoretical Prospects of Neutrinoless Double Beta Decay", hep-ph/0504166

[24] S. Choubey, W. Rodejohann, Phys. Rev. D 72, 033016
(2005).

[25] A. G. Dias, A. Doff, C. A. deS. Pires and P. S. Rodrigues da Silva, Phys. Rev. D 72, 035006 (2005).

[26] S. M. Bilenky, A. Faessler, T. Gutsche and F. Simkovic, Phys.Rev. D 72, 053015 (2005).

[27] Heidelberg-Moscow collaboration, H. KlapdorKleingrothaus et al, Nulc. Phys. Proc. Suppl. 110, 364 (2002); Phys. Lett. B586, 198 (2004).

[28] G. G. Raffelt, "Astrophysical and cosmological neutrinos", hep-ph/0208024

[29] S. Elliott and P.Vogel, Ann. Rev. Nucl. Part. Sci. 52, 115 (2002).

[30] S. Eidelman et al, "Review of particle physics" (Particle data group), Phys. Lett. 592, 1 (2004), http://pdg.lbl.gov

[31] G. Altarelli and F. Feruglio, New J. Phys. 6, 106 (2004).

[32] Ch. Kraus et al, Eur.Phys.J. C40, 447 (2005).

[33] A. Yu. Smirnov, Int. J. Mod. Phys. A19,1180 (2004).

[34] R. N. Mohapatra et al, "Theory of Neutrinos: A White Paper", hep-ph/0510213

[35] M. Gell-Mann, P. Ramond and R. Slansky, "Complex spinors and unified theories", in "Supergravity", P. van Nieuwenhuizen and D. Friedman, eds., North Holland, Amsterdam, 1979, p. 315; R. N. Mohapatra and G. Senjanović, Phys. Rev. Lett. 44, 912 (1980).

[36] R. N. Mohapatra and P. B. Pal, "Massive neutrinos in physics and astrophysics", World Scientific, Singapore 1998.

[37] F. Boehm and P. Vogel, "Physics of massive neutrinos", Cambridge university press, Cambridge 1992.

[38] P. Langacker, "Neutrino physics", hep-ph/0506257

[39] A. Yu. Smirnov and M. Tanimoto, Phys. Rev. D 55, 1665 (1997); A. Yu. Smirnov, "Alternatives to the seesaw mechanism", hep-ph/0411194

[40] S. M. Bilenky and S. T. Petcov, Rev. Mod. Phys. 59, 671 (1987); Erratum: ibid. 60, 575 (1988); ibid. 61, 169 (1989).

[41] Nguyen Tuan Anh, Nguyen Anh Ky and Hoang Ngoc Long, Int. J. Mod. Phys. A15, 283 (2000); ibid. A16, 541 (2001).

[42] Nguyen Anh Ky, "Comments related to reading "Static quantities of the $W$ boson in the $S U_{L}(3) \times U_{X}(1)$ model with right-handed neutrinos", hep-ph/0504147

[43] M. Singer, J. W. F. Valle and J. Schechter, Phys. Rev. D 22, 738 (1980).

[44] J. W. F. Valle and M. Singer, Phys. Rev. D 28, 540 (1983).

[45] J. C. Montero, F. Pisano and V. Pleitez, Phys. Rev. D 47, 2918 (1993). 